\title{
Theoretical Approach to Simulate Efficient Selective Solar Absorbers With Micro or Nano Structured Arrays
}

\author{
Jhong-sian Chiu', Wen-Bin Young ${ }^{a} * \mathbb{B}$ \\ ${ }^{a}$ Department of Aeronautics and Astronautics, National Cheng-Kung University, Tainan, Taiwan
}

Received: November 21, 2017; Revised: February 01, 2018; Accepted: February 27, 2018

\begin{abstract}
Study of the solar absorber with photonic crystal or micro structures as the heat absorbing surfaces operated at a high-temperature was conducted. Numerical calculations based on rigorous coupled-wave analysis were performed to determine the absorptance of the photonic crystal surface on the absorber. The effect of nano geometry of the photonic crystal in tungsten on the selectivity of the absorber was investigated first, including period (L), aperture (A) and depth (d). A design algorithm for the photonic crystal heat absorbing surface was summarized. Based on the results, a design with combined large and small square patterns was proposed to improve the absorptance, which demonstrated the ability to broaden the absorbing band by mix-period nano patterns. Integration of an anti-reflection coating (ARC) on the photonic crystal using aluminum oxide $\left(\mathrm{Al}_{2} \mathrm{O}_{3}\right)$ was also studied. It was shown that, under a specific thickness of $\mathrm{ARC}$, the absorptance can be further improved. As considering the effect of concentration coefficient on the energy absorption efficiency, it was found that a micro structure fabricated by laser ablation on stainless steel has a conversion rate over $85 \%$ in the case using a high concentrate coefficient.
\end{abstract}

Keywords: Selective solar absorber, photonic crystal, nano/micro structure, dish concentrating solar power.

\section{Introduction}

Design of a high efficiency solar absorber is important in the concentrating solar power system. The primary requirements for the absorber are having a high heat absorptance at the solar spectrum region, while keeping low absorptance at the black body emittance region corresponding to the working temperature. According to the usage of structures and materials, there are six major designs of selective solar absorbers being reported in literature ${ }^{1}$ : (a) intrinsic absorbers, (b) semiconductor-metal tandems, (c) multilayer absorbers, (d) Cermets absorbers, (e) surface texturing, and (f) photonic crystals (PhC) based absorbers. Intrinsic absorbers use intrinsic properties of the material to obtain desired spectrum, so its performance is limited by the material itself. Semiconductor-metal tandems use the properties of semiconductors with an appropriate bandgap to absorb short wavelength radiation, and keep high reflectance in the infrared region. The major drawback of it is being not stable at the temperature higher than $750^{\circ} \mathrm{C}^{2}$. Both of multilayer absorbers and cermets absorbers combine the metals and dielectrics as the structure to have good ability to adjust the desired absorbing spectrum. These materials have good performance at the mid-temperature range up to the temperature about $700{ }^{\circ} \mathrm{C}^{3-5}$. The fabrication of surface texturing is accomplished by surface roughening technology. It traps the light of short wavelength in the texture of the rough surface and reflects the light in longer wavelength ${ }^{6,7}$. The last design is photonic crystals $(\mathrm{PhC})$ installed on the heat absorbing surface of the absorber. The absorbing spectrum of $\mathrm{PhC}$ can be well controlled by varying the geometry sizes of the nano structures. The $\mathrm{PhC}$ can be installed on a surface of a heat-resistant material having good thermal stability at a high-temperature as tungsten (W) or tantalum (Ta).

In literature, there are many studies on optical properties of photonic crystals concentrated on investigating the effects of nano structures including period, aperture, depth and incident angle ${ }^{8-11}$ on the absorbing spectrum. The period of the nano structure determines the lower bound wavelength of the absorbing band in a $0^{\circ}$ incident angle. As the depth of the nano structure increases, the absorptance in the absorbing band region shows some periodic behavior. Increasing the depth or the aperture of $\mathrm{PhC}$ structure can also increase the absorptance in absorbing band region. The variation of the aperture will also alter the position of the cut-off wavelength, which is approximately corresponding to the upper bound wavelength of the absorbing band.

In the recent years, literature showed that coating a material as anti-reflection coating (ARC) with refractive index between the index of air and the structure on the absorber could reduce the effect of the incident angle. It was shown that the emissivity spectrum of the tungsten (W) cavity structure filled with the dielectric material with refractive index $n=1.8$ can reduce the effect of the incident angle from $0^{\circ} \sim 45^{\circ 12}$. There are also some reports ${ }^{8,9,13-16}$ showing that coating a thin film of $\mathrm{Al}_{2} \mathrm{O}_{3}$ or $\mathrm{HfO}_{2}$ on the structure of $\mathrm{W}$ or Ta can improve the resistant to high temperature and also the absorptance as well. In the actual operation, the thin film coated on nano structure can also serve as a protection layer keeping structure from damage. 
In this study, numerical calculations based on rigorous coupled-wave analysis were used to determine the absorptance of the photonic crystal surface in the absorber. The effect of nano geometry of the photonic crystal on the selectivity of the absorber was investigated, including period (L), aperture (A) and depth (d). Based on the results, a design with combined large and small square patterns was proposed to improve the absorptance, which demonstrated the ability to broaden the absorbing band by mix-period nano patterns. Integration of an anti-reflection coating on the photonic crystal using aluminum oxide $\left(\mathrm{Al}_{2} \mathrm{O}_{3}\right)$ was also studied. The effect of concentration factor on the absorption efficiency was also discussed, which leaded to the possible application of micro structure design fabricated by laser ablation on the surface of the stainless steel solar absorber.

\subsection{Selective absorber}

The average solar absorptance $\bar{a}$ and thermal emissivity $\bar{\varepsilon}$ of the absorber can be calculated from reflectance spectrum, and are defined as:

$$
\begin{gathered}
\bar{\alpha}=\frac{\int_{0}^{\infty}[1-R(\lambda)] G(\lambda) d \lambda}{\int_{0}^{\infty} G(\lambda) d \lambda} \\
\bar{\varepsilon}(T)=\frac{\int_{0}^{\infty}[1-R(\lambda)] B(\lambda, T) d \lambda}{\int_{0}^{\infty} B(\lambda, T) d \lambda}
\end{gathered}
$$

Where $R(\lambda)$ is the reflectance, $\lambda$ is the wavelength of light, $G(\lambda)$ is the solar spectrum of AM1.5 $5^{17}, T$ is the operating temperature, and $B(\lambda, T)$ is the intensity of blackbody radiation spectrum at temperature $T$.

As a figure of merit, the solar photothermal conversion coefficient $\eta_{\text {FOM }}^{*}$, which is a ratio of the total absorbed energy to the energy delivered, is defined as:

$$
\eta *_{F O M}=\frac{C * \int_{0}^{\infty}[1-R(\lambda)] G(\lambda) d \lambda-\int_{0}^{\infty}[1-R(\lambda)] B(\lambda, T) d \lambda}{C^{*} \int_{0}^{\infty} G(\lambda) d \lambda}
$$

where $C$ is the concentration factor. The conversion coefficient $\eta_{\text {FOM }}^{*}$ can be rearranged as following form:

$$
\begin{gathered}
\eta *_{F O M}=\bar{\alpha}-\frac{1}{C} * M(T) \\
M(T)=\frac{\int_{0}^{\infty}[1-R(\lambda)] B(\lambda, T) d \lambda}{\int_{0}^{\infty} G(\lambda) d \lambda}
\end{gathered}
$$

where $M(T)$ is the term represented the influence of thermal radiation and can be considered as the thermal radiation factor. In equation (4), increasing the concentration factor $C$ can reduce the effect of thermal radiation factor $M(T)$, and that will increase the conversion coefficient. Thus, if $M(T)$ is very small or $C$ is very large, the photothermal conversion efficiency is nearly proportional to the average absorptance $\bar{a}$.

An ideal solar absorber should have high absorptance in the solar spectrum while having low emissivity in the thermal radiation spectrum. The ideal spectral absorptance of a selective absorber will have a cut-off wavelength defined by the intersection of the concentrated solar radiation and black body emission spectra. It must be noticed that the intensity of the concentrated solar radiation depends on the concentration factor. As the concentration factor increases, the intensity of concentrated solar radiation increases also, resulting in a larger cut-off wavelength.

\subsection{Simulation of $R C W A$}

Rigorous coupled-wave analysis (RCWA) is a method for modeling the diffraction of electromagnetic waves on the periodic grating array. It solves Maxwell's equation corresponding to the light scattering problem by using a Fourier expansion to represent the optical property at periodic grating region based on the Floquet's theorem. Since the solution is represented by infinite Fourier expansion series, a truncation of the expansions is necessary in evaluation of the solution. Selection of the number of Fourier terms becomes the main issue related to the accuracy of the solution ${ }^{18-23}$. In this study, we followed the RCWA formulation and solution in literature ${ }^{21}$, and considered the diffraction order up to \pm 24 th terms for both $\mathrm{X}$ and $\mathrm{Y}$ directions.

As shown in Fig. 1(a), we simulated the reflectance spectra of periodic cavity array at normal incidence, so the incident angles $\theta$, the azimuthal angle $\phi$, and the polarization angle $\Psi$ are equal to $0^{\circ}$, and both the periods in $\mathrm{X}$ and $\mathrm{Y}$ direction are equal to $\mathrm{L}$. The material used in this study for $\mathrm{PhC}$ was tungsten. We also studied the photonic crystal deposited with high step coverage $\mathrm{ARC}$ using $\mathrm{Al}_{2} \mathrm{O}_{3}$. The cross section view of a $\mathrm{PhC}$ with ARC is shown in Fig 1(b) where $\mathrm{d}$ is the depth of the $\mathrm{PhC}$ pattern and $t$ is the thickness of ARC. All the optical properties used in this study were referred from literature where those were measured at room temperature ${ }^{24}$ and listed in Table 1.

Table 1. The refractive index at the wavelength of $0.667 \mu \mathrm{m}$

\begin{tabular}{lccc}
\hline & tungsten & $\mathrm{Al}_{2} \mathrm{O}_{3}$ & air \\
\hline Refractive index & 3.8313 & 1.7644 & 1 \\
Extinction coefficient & 2.9043 & --- & --- \\
\hline
\end{tabular}

\section{Result and Discussion}

Two nano patterns with circular or square cavities for $\mathrm{PhC}$ were included in the study. The aperture of cavity (A) is the opening of the unit cell in the cavity pattern. In the unit cell, a filling ratio $(\mathrm{F})$ is defined as the ratio of the period (L) divided by the aperture. In the case of circular cavity, the aperture is equal to the diameter.

\subsection{Simulation model validation}

For validating the simulations in this study, we simulated the periodic circular or square cavity structures which were the same geometry as in literature ${ }^{10,12}$. The nano pattern with circular cavities had the dimensions of period $\mathrm{L}=0.7$ $\mu \mathrm{m}$, aperture $\mathrm{A}=0.6 \mu \mathrm{m}$, and depth $\mathrm{d}=1.2 \mu \mathrm{m}$ using the tungsten as the substrate material, and the cavity was filled with a material in a refractive index (n) of 1.8 as used in 
literature. In the case of periodic pattern with square cavities, the model had periods varied from $0.4 \mu \mathrm{m}$ to $0.6 \mu \mathrm{m}$, and the aperture $\mathrm{A}=0.8 \mathrm{~L}$ and the depth $\mathrm{d}=\mathrm{A}$. Fig. 2 shows the comparisons of simulation results between literature and our simulation. The simulation results for both cases with either circular or square cavities agreed quite well with literatures, which demonstrated the validity of our simulation.

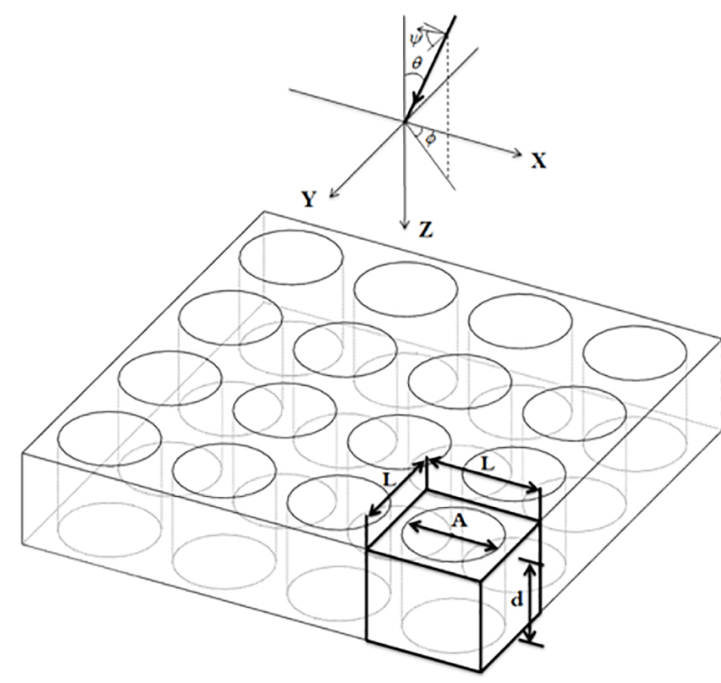

(a)

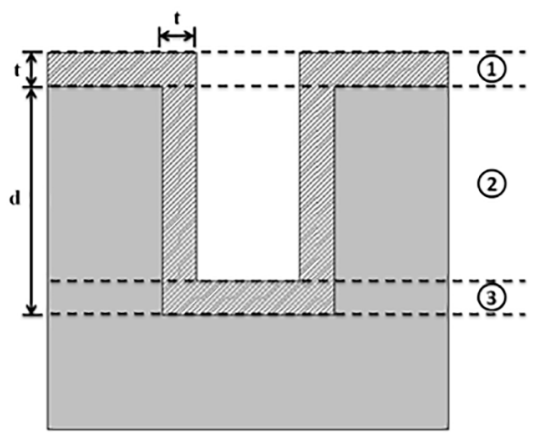

(b)

Figure 1. (a) Schematic diagram of PhC model for RCWA calculations (b) photonic crystals deposited with high step coverage ARC

\subsection{Influence of geometry of the photonic crystal}

In the following, we investigate the effect of period, depth, and aperture of $\mathrm{PhC}$ structure on reflectance spectrum. This study used circular cavity pattern with the dimensions of the period $\mathrm{L}=1.6 \mu \mathrm{m}$ and the aperture $\mathrm{A}$ varied from $1 \mu \mathrm{m}$ to $1.4 \mu \mathrm{m}$. The depth was also changed from $0.05 \mu \mathrm{m}$ to $10 \mu \mathrm{m}$.

The simulation reflectance spectrum is shown in Fig. 3 for PhCs with the same period and different apertures of 1.0,1.2, and $1.4 \mu \mathrm{m}$. The reflectance spectra of PhCs with different depths were also shown in the figure. For $1.0 \mu \mathrm{m}$ aperture in Fig. 3(a), the reflectance

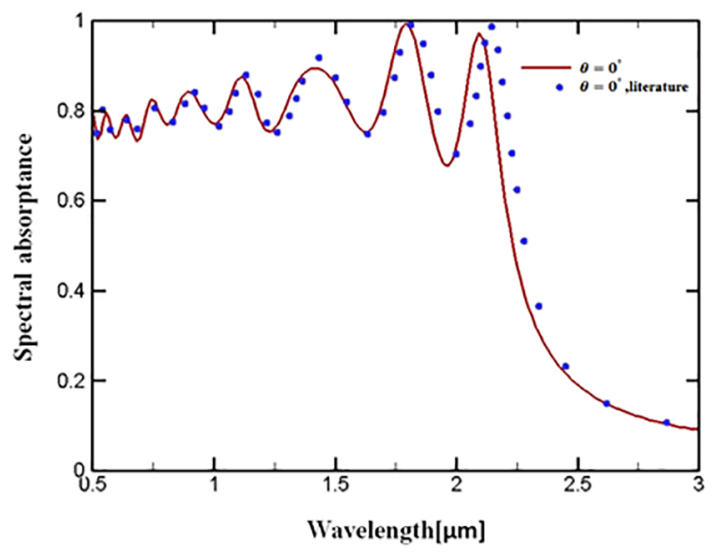

(a)

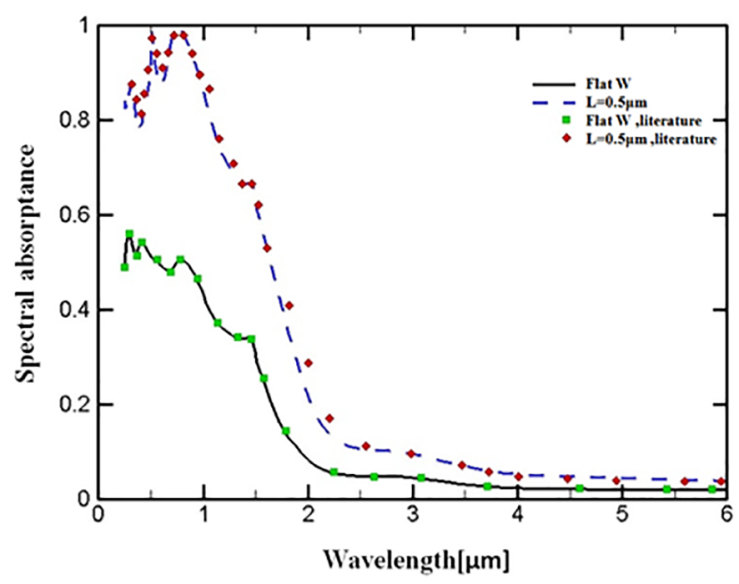

(b)

Figure 2. Absorptance spectra from literature ${ }^{(10)}$ and current simulation for (a) nano circular pattern (b) flat tungsten and nano square pattern.

spectrum shows one clear trough at the position with the wavelength near the scale of the period. The reflectance at the trough of the spectrum decreases with increase of the cavity depth and saturates beyond $3 \mu \mathrm{m}(\mathrm{d} / \mathrm{A}=3)$, indicating that, beyond this maximum depth, it will not cause any change in the reflectance spectra.

For cases with larger apertures of $\mathrm{A}=1.2 \mu \mathrm{m}$ or $1.4 \mu \mathrm{m}$, the resulting reflectance spectra are shown in Fig. 3(b) and 3(c). In the two figures, a low reflectance band shows up for the case when depth is larger the ratio of $\mathrm{d} / \mathrm{A}$. The reflectance has some wavy variation at this band, and larger depth results in lower reflectance in general. The amplitude of waviness decreases with increase of the depth also. In addition, the spectra show that the reflectance decreases with the increase of depth as well. It was concluded that, as the aperture ratio of $\mathrm{A} / \mathrm{L}$ is greater than 0.7 , the reflectance spectrum will has a low reflectance band ranging from $\mathrm{L}$ to $2 \mathrm{~A}$. 


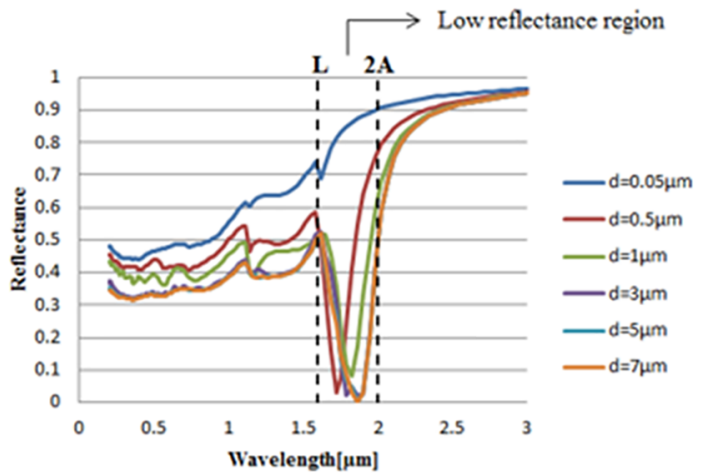

(a)

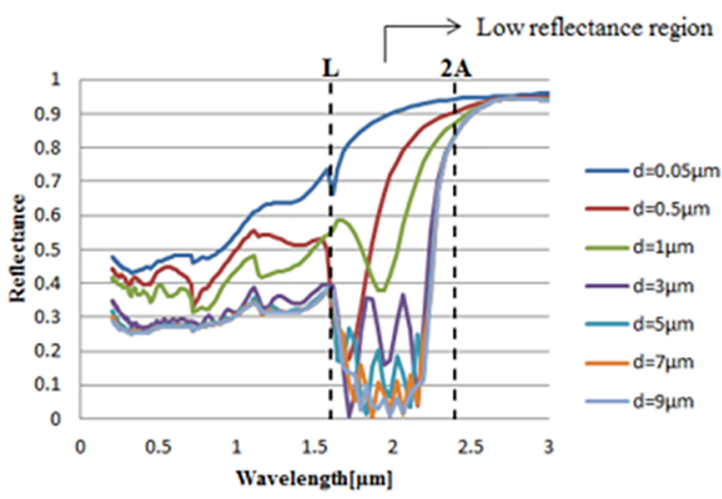

(b)

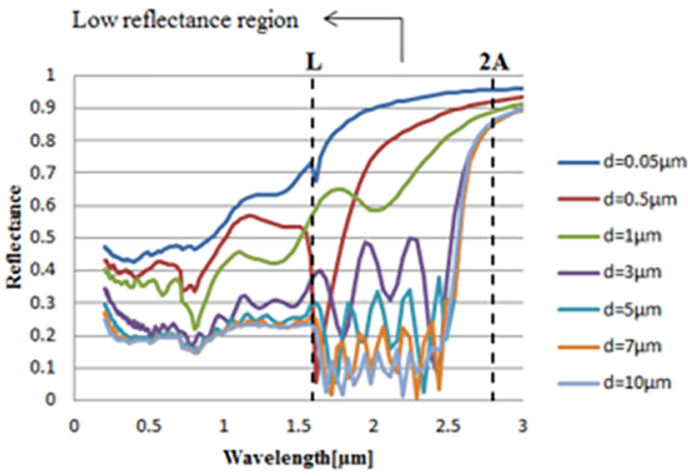

(c)

Figure 3. Reflectance spectrum for different depths of circular pattern in $\mathrm{PhC}$ with a period $\mathrm{L}=1.6 \mu \mathrm{m}$ and aperture $\mathrm{A}=(\mathrm{a}) 1 \mu \mathrm{m}$ (b) $1.2 \mu \mathrm{m}$ (c) $1.4 \mu \mathrm{m}$.

In order to show the influence of period of photonic structure on the reflectance spectrum, nano structures with different periods $(1.25 \sim 5 \mu \mathrm{m})$ was simulated with the aperture defined in two cases as $\mathrm{A}=1 \mu \mathrm{m}$ and $\mathrm{A}=\mathrm{L}-0.6 \mu \mathrm{m}$ respectively. The depth of the structure remains equaling to the period. Fig. 4 shows the resulting reflectance spectra of the $\mathrm{PhCs}$ together with the case of flat tungsten. Notice that all the spectra have a low reflectance trough at the wavelength close to period (indicated in black dash line). The result indicates that the period of the PhC structure primarily determines the region of low reflectance in the spectrum.

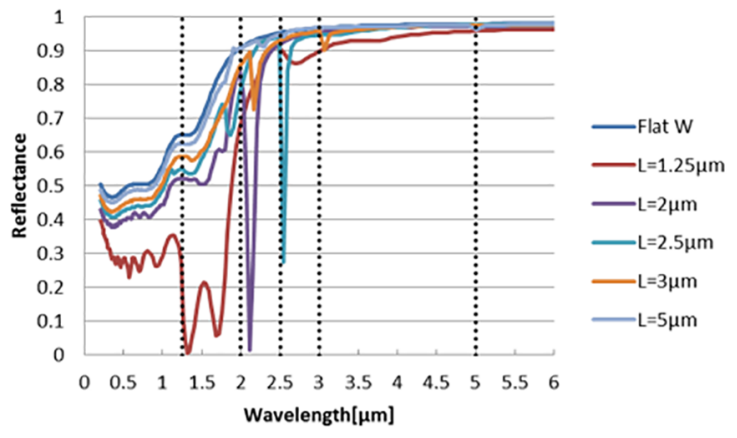

(a)

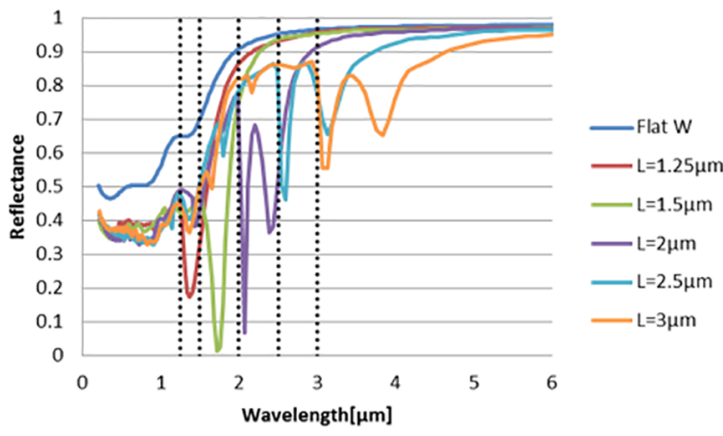

(b)

Figure 4. Reflectance spectrum for different periods (L) with (a) $\mathrm{A}=1 \mu \mathrm{m}$ (b) $\mathrm{A}=\mathrm{L}-0.6 \mu \mathrm{m}$ and depth $\mathrm{d}=\mathrm{A}$.

The reflectance spectra for the cases with fixed aperture of $\mathrm{A}=1 \mu \mathrm{m}$ are shown in Fig. 4(a). Under this circumstance, the aperture ratio of aperture to period will decrease with increasing period. Following the condition discussed above, the aperture ratio must be larger than 0.7 in order to have a low reflectance band. As sown in the figure, only the case with $\mathrm{L}=1.25 \mu \mathrm{m}$ fulfills this condition and shows low reflectance band in the spectrum. For larger periods, there is only a sharp decrease in reflectance at the wavelength equaling to the period. For the situation of A $=\mathrm{L}-0.6 \mu \mathrm{m}$, the aperture to period ratio increases with the period. Therefore, as the ratio is larger than 0.7 (i.e. $\mathrm{L}>2 \mu \mathrm{m}$ ), the spectrum will have a low reflectance band as shown in Figure 4(b).

Because of the ideal cut-off wavelength changing with different operating condition, the best absorber suited for all conditions does not exist. Thus, in the following we will briefly narrate how to design a better absorber according to the result above.

At first, identify the range of wavelength which we want to absorb and the position of ideal cut-off wavelength. Then define the smallest wavelength in that range as the period of $\mathrm{PhC}$ structure so that the corresponding spectra will have high absorptance starting at that wavelength. With regard to solar absorbers, the better period of $\mathrm{PhC}$ structure is about $0.4 \mu \mathrm{m}$ $\sim 0.5 \mu \mathrm{m}$. To generate a wider high absorptance band, a larger aperture ratio is required. However, due to the limitation of 
the fabrication process, an aperture can be selected within this limit. Then, one can increase the depth until it has no further effect on the reflectance spectrum. If the band extends beyond the cut-off wavelength, reduce the aperture and do the study on depth again. After these procedures, one can have a photonic crystal absorber with high efficiency.

\subsection{Nano geometry of PhC}

In applying $\mathrm{PhC}$ as the solar absorber, the patterned structures in either circular or square cavity are commonly found in literature. A comparison on $\mathrm{PhC}$ patterned with these two geometries was made to understand their effect on the solar absorptance. Simulations were performed with the parameters of $\mathrm{L}=0.4 \mu \mathrm{m}$ and $\mathrm{d}=0.32 \mu \mathrm{m}$ with different filling ratios. The filling ratio is defined as the ratio of the aperture to period. The calculated average absorptance of $\mathrm{PhC}$ with patterned structure in square or circular cavity is shown show in Fig. 5. It is apparent that $\mathrm{PhC}$ with square cavity patterned structure will have higher absorptance as compared to the circular cavity. The absorptance also increases with the filling ratio as discussed above. With this result, the nano structure in square cavity pattern will be used for further discussion. The average absorptance and emittance of type $1 \mathrm{PhC}$ were listed in Table 2.

Table 2. The average absorptance and emittance of type $1 \mathrm{PhC}$

\begin{tabular}{lccccc}
\hline \multirow{2}{*}{ Type1 } & $\begin{array}{c}d= \\
0.32 \mu m\end{array}$ & $\begin{array}{c}d= \\
0.64 \mu m\end{array}$ & $d=1 \mu m$ & $\begin{array}{c}d= \\
1.5 \mu m\end{array}$ & $d=2 \mu m$ \\
\hline $\bar{\alpha}$ & 0.8126 & 0.8273 & 0.8282 & 0.8284 & 0.8284 \\
$\bar{\varepsilon}(1073 \mathrm{~K})$ & 0.1015 & 0.1055 & 0.1055 & 0.1055 & 0.1055 \\
$\bar{\varepsilon}(1500 \mathrm{~K})$ & 0.1982 & 0.2052 & 0.2053 & 0.2053 & 0.2053 \\
\hline
\end{tabular}

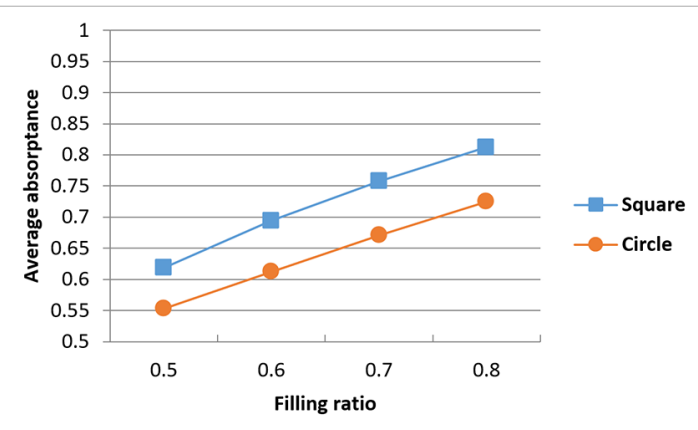

Figure 5. Average absorptance of $\mathrm{PhC}$ with patterned square or circular structures in different filling ratios.

\subsection{Mix-period photonic crystal}

The PhC analyzed so far has the nano structure with the same period. Combination of mix-period design will be considered in this section. The $\mathrm{PhC}$ with the same period of $\mathrm{L}=0.4 \mu \mathrm{m}$ and aperture of $\mathrm{A}=0.8 \mathrm{~L}$ was defined as type 1 as shown in Fig. 6. Two kinds of mix-period patterns were designed as type 2 and 3 as well in the figure. RCWA was performed on three types of $\mathrm{PhC}$. Through the comparisons of these three types, the influence of mix-period on absorptance spectra in solar absorber was investigated. In Fig. 7(a), the absorptance spectra of three types show that mix-period pattern results in wider range of high absorptance band than single-period pattern. This may be caused by the superposition of the high absorptance regions corresponding to different periods. The selected depth of the patterned structure was $2 \mu \mathrm{m}$ as further increase of the depth did not change the absorptance. The design of type 3 is superior to the others as it has an apparently wider high absorptance band. The average absorptance and emittance of type 2 and $3 \mathrm{PhC}$ were listed in Table 3 and 4.

Table 3. The average absorptance and emittance of type $2 \mathrm{PhC}$

\begin{tabular}{lccccc}
\hline Type2 & $\begin{array}{c}\boldsymbol{d}= \\
0.32 \boldsymbol{\mu m}\end{array}$ & $\boldsymbol{d}=1 \boldsymbol{\mu m}$ & $\begin{array}{c}\boldsymbol{d}= \\
1.5 \boldsymbol{\mu m}\end{array}$ & $\boldsymbol{d}=2 \boldsymbol{\mu m}$ & $\boldsymbol{d}=3 \boldsymbol{\mu m}$ \\
\hline $\bar{\alpha}$ & 0.8374 & 0.8565 & 0.8565 & 0.8565 & 0.8565 \\
$\bar{\varepsilon}(1073 \mathrm{~K})$ & 0.1239 & 0.1318 & 0.1318 & 0.1318 & 0.1318 \\
$\bar{\varepsilon}(1500 \mathrm{~K})$ & 0.2365 & 0.2487 & 0.2487 & 0.2487 & 0.2487 \\
\hline
\end{tabular}

Table 4. The average absorptance and emittance of type $3 \mathrm{PhC}$

\begin{tabular}{lcccc}
\hline Type 3 & $\begin{array}{c}\boldsymbol{d}= \\
0.32 \boldsymbol{\mu m}\end{array}$ & $\boldsymbol{d}=1 \boldsymbol{\mu m}$ & $\boldsymbol{d}=2 \boldsymbol{\mu m}$ & $\boldsymbol{d}=3 \boldsymbol{\mu m}$ \\
\hline $\bar{\alpha}$ & 0.8074 & 0.8820 & 0.8973 & 0.8975 \\
$\bar{\varepsilon}(1073 \mathrm{~K})$ & 0.1289 & 0.1723 & 0.1746 & 0.1746 \\
$\bar{\varepsilon}(1500 \mathrm{~K})$ & 0.2442 & 0.3166 & 0.3220 & 0.3221 \\
\hline
\end{tabular}

The $\mathrm{PhC}$ with type 3 mix-period design was used to study the effect of deposited anti-reflective coating (ARC). The material of $\mathrm{ARC}$ used was aluminum oxide $\left(\mathrm{Al}_{2} \mathrm{O}_{3}\right)$, and its optical constants were derived from the literature ${ }^{24}$. The first case was to investigate the influence of thickness of ARC on the absorptance spectrum. The calculated absorptance spectra of $\mathrm{PhC}$ structures with different thickness of ARC from 0.03 to $0.07 \mu \mathrm{m}$ are shown in Fig. 7(b). The absorptance of the high absorptance band increases with the ARC thickness as shown in the figure. The absorptance band only shows a shift to the right hand side with the increase of ARC thickness. Therefore, the cut-off wavelength of the absorptance spectrum increases with the ARC thickness. If one looks into the solar irradiation spectrum, the radiation intensity rises rapidly at the wavelength of $0.3 \mu \mathrm{m}$, and reaches the peak at around $0.5 \mu \mathrm{m}$. Therefore, for application on the solar absorber, the absorptance spectrum of $\mathrm{PhC}$ should be designed to have the high absorptance band to start from $0.3 \mu \mathrm{m}$ to the cut-off wavelength.

The other case was to investigate the influence of the depth of type 3 structure with ARC coating. The absorptance spectra were shown in Fig. 7(c) for $\mathrm{PhC}$ with different structure depths from 0.32 to $2 \mu \mathrm{m}$ and ARC coating in $0.07 \mu \mathrm{m}$ thickness. The bandwidth of high absorptance band increases with the depth of the type 3 structure, while the starting point of the band did not change. The cut-off wavelength increases with the depth correspondently. 
Type 1

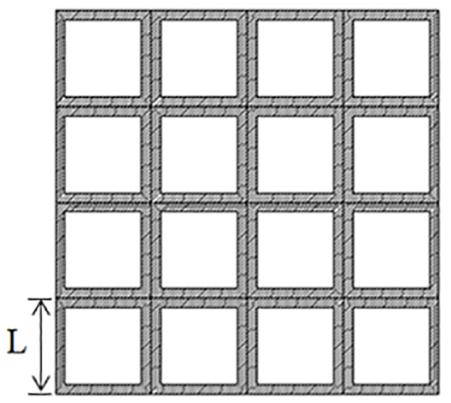

Type 2

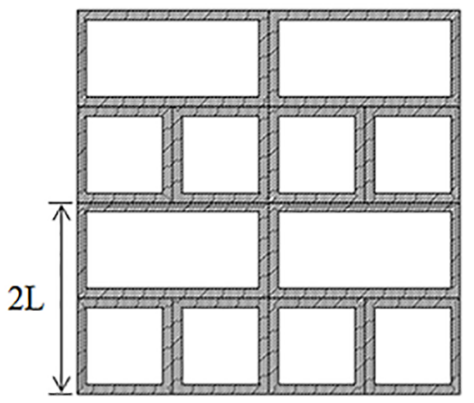

Type 3

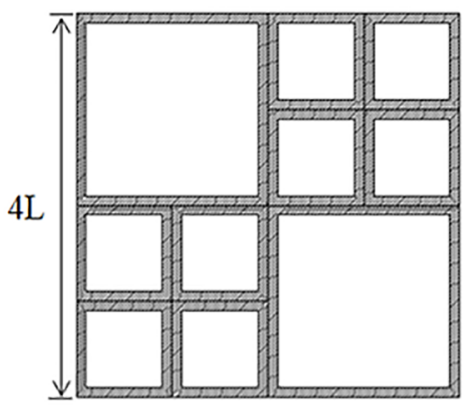

Figure 6. Top views of three nanostructure types.

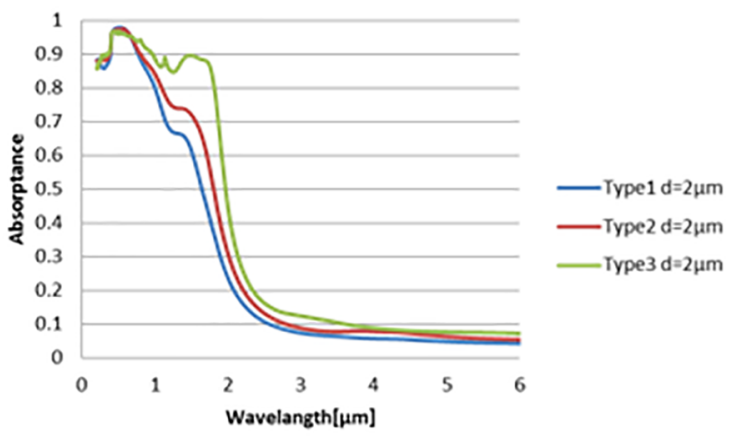

(a)

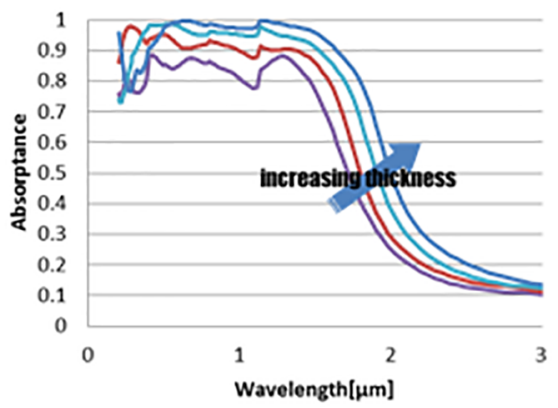

(b)

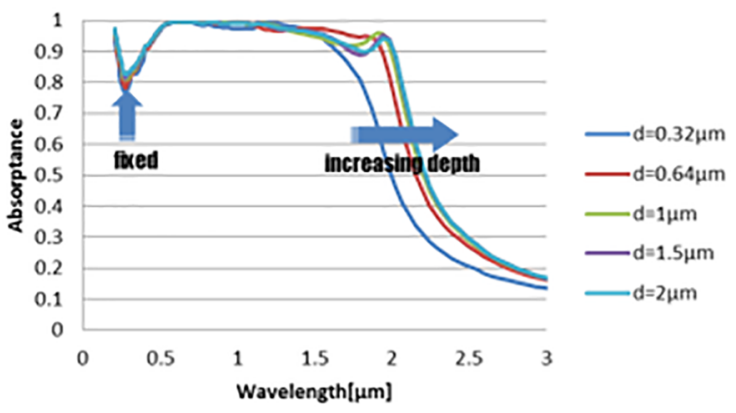

(c)

Figure 7. Absorptance spectrum of (a) different $\mathrm{PhC}$ types (b) type $3 \mathrm{PhC}$ with different ARC thickness coatings (c) type $3 \mathrm{PhC}$ with different structure depths and ARC coating of $0.07 \mu \mathrm{m}$.
To further understand the effect of the type 3 structure depth on the thermal absorption, calculation the photothermal conversion coefficient was done in two operating temperatures. The photothermal conversion coefficient $\left(\eta_{\text {FOM }}^{*}\right)$ is a function of temperature and concentration factor as shown in Eq. 3. The ideal cut-off wavelengths for black body radiation at temperature $\mathrm{T}=1073 \mathrm{~K}$ or $\mathrm{T}=1500 \mathrm{k}$ are around $2.47 \mu \mathrm{m}$ and $1.78 \mu \mathrm{m}$ respectively. Fig. 8 shows the $\eta_{\text {FoM }}^{*}$ of the type 3 structure with different depths under two different temperatures. As shown in Fig. 7(c), the absorptance band for type 3 structure was located about from 0.3 to 2.2 $\mu \mathrm{m}$. At temperature $\mathrm{T}=1073 \mathrm{~K}$, the cut-off wavelength $(2.47 \mu \mathrm{m})$ is larger than the band wavelength for the type 3 structures at different depths. Therefore, $\eta^{*}{ }_{\text {FOM }}$ increases with the structure depth. On the other hand, for temperature $\mathrm{T}=1500 \mathrm{~K}$, the cut-off wavelength $(1.78 \mu \mathrm{m})$ is inside the absorptance band at the larger structure depth. The conversion coefficient decreases with the structure depth.

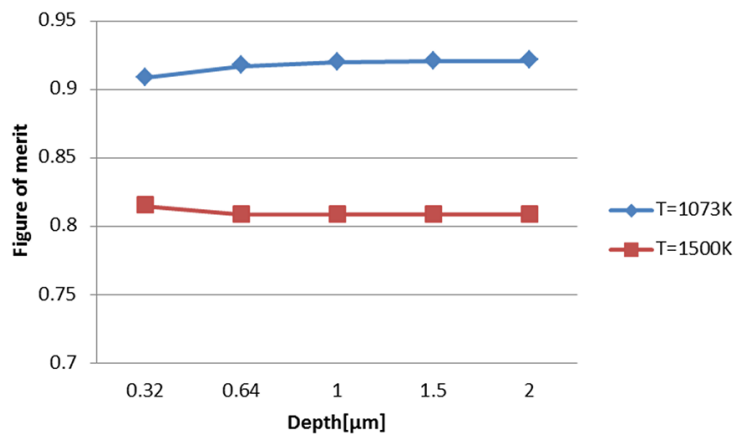

Figure 8. Figure of merit for increasing depth at temperatures $\mathrm{T}=1073 \mathrm{~K}$ or $\mathrm{T}=1500 \mathrm{~K}$.

\subsection{Photothermal conversion Efficiency analysis}

As discussed above about the photothermal conversion coefficient, it depends not only on the selective absorptance spectrum of the absorber but also on the operating temperature. The dependence of the conversion coefficient on the temperature is through the effect of concentration factor as shown in Eq. 4. In order to understand the effect, a material with perfect absorptance is assumed. The photothermal conversion coefficient was calculated 
at temperatures of $873 \mathrm{~K}, 1073 \mathrm{~K}, 1273 \mathrm{~K}$ and $1473 \mathrm{~K}$ under different concentration factors as shown in Fig. 9. The conversion coefficient decreases with the increase of operating temperature under a fixed concentration factor. However, the photothermal conversion coefficient can reach $90 \%$ with a high concentration factor of 1000 at the temperature less than $1073 \mathrm{~K}$. The result means that, as long as the absorptance of the absorber is high enough, it still can achieve high efficiency of photothermal conversion without the selective absorptance spectrum.

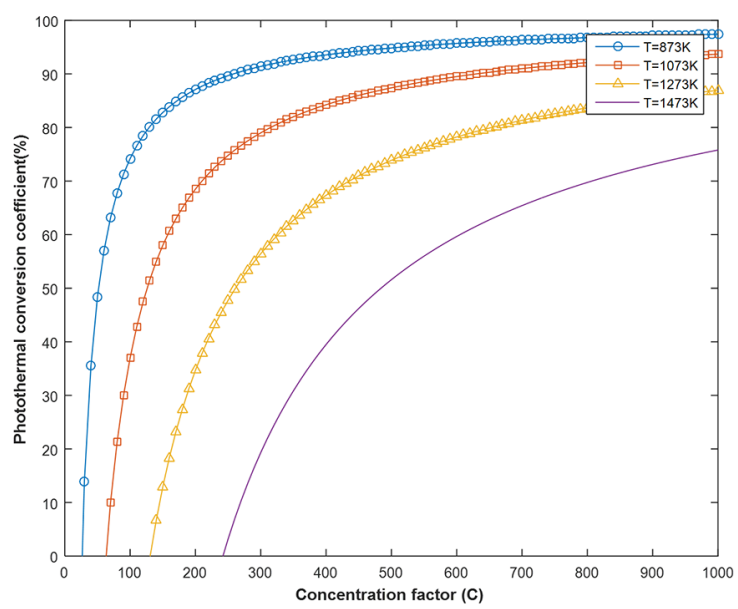

Figure 9. Photothermal conversion coefficient for different concentration factors and operating temperatures.

\subsection{Micro structure}

Based on the conclusion above, an absorber with high average absorptance can have photothermal conversion up to 0.9 at the temperature below $1073 \mathrm{~K}$. In order for comparison of the performance of micro structures with respect to $\mathrm{PhC}$, an absorber was fabricated to improve the absorptance by constructing micro scale structures on stainless steel by laser ablation using a laser engraving machine. Parallel straight channels were cut on the surface of stainless steel. The laser ablation speed was $5 \mathrm{~mm} / \mathrm{sec}$, the pitch was $0.04 \mathrm{~mm}$, and the frequency was $20 \mathrm{kHz}$.

A spectrum analyzer was used to measure the reflectance in the wavelength from $0.25 \mu \mathrm{m}$ to $2.5 \mu \mathrm{m}$, while a Fourier Transform infrared spectroscopy was used in the wavelength from $2.5 \mu \mathrm{m}$ to $15 \mu \mathrm{m}$. The derived absorptance spectra of the absorber ablated with different laser power at 10 $\mathrm{W}, 14 \mathrm{~W}$ and $18 \mathrm{~W}$ respectively are shown in Fig. 10 . The absorptance increases with increase of laser ablation power. In the range of visible and near infrared region, the absorptance can reach $0.85 \sim 0.96$ for the sample made in power of $18 \mathrm{~W}$. However, no selective band and apparent cut-off wavelength were found in the spectra.

Then the $\eta_{\text {FOM }}^{*}$ of the micro structure fabricated by laser power $18 \mathrm{~W}$ was calculated to compare with the type 3 nano structure with ARC $(\mathrm{t}=0.07 \mu \mathrm{m})$ in Fig 11. The spectrum of flat tungsten at $\mathrm{T}=1073 \mathrm{~K}$ was also included. The dash lines are the average absorptance of these three absorbers respectively, and the efficiency can be found to increase with the concentration factor. When the operating condition is at $\mathrm{C}=1000$, the $\eta_{\text {FOM }}^{*}$ of the micro structure is about 0.87 , which has made some improvement as compared to the flat tungsten. The efficiency can be further improved by using a design with a higher concentration factor.

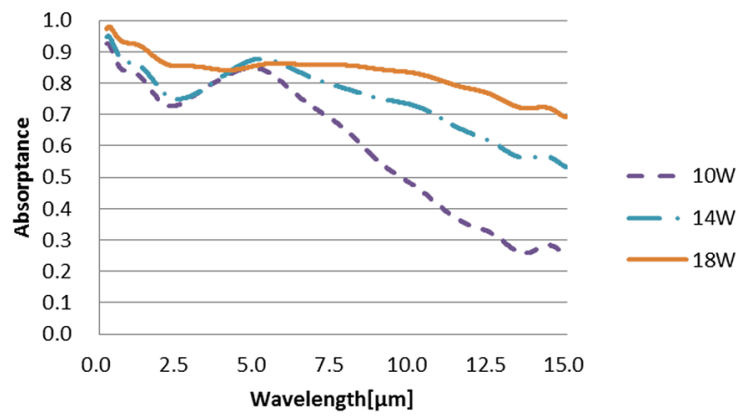

Figure 10. Absorptance spectrum of SS engraved by different power of laser.

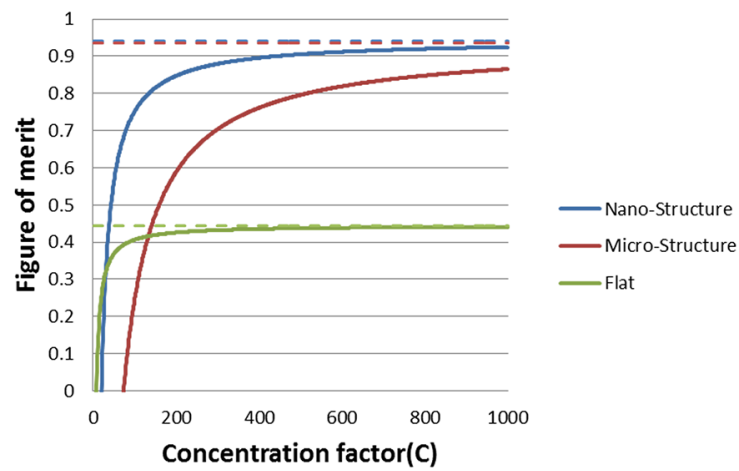

Figure 11. Figure of merit for three kinds of absorbers.

\section{Conclusion}

Photonic crystal is a good candidate as the absorbing surfaces of a solar absorber. The effect of the geometric parameters of $\mathrm{PhC}$ on its selective spectra was studied. The lower bound of the selective band (high absorptance) was found to near the period of the $\mathrm{PhC}$ structure and the upper bound was the cut-off wavelength. Larger aperture and depth of the $\mathrm{PhC}$ structure were preferred to have wider selective band. It was also demonstrated that the $\mathrm{PhC}$ with mix period nanostructure has a wider range of selective band than the single period. Based the analysis of photothermal conversion coefficient, absorber without selective band operated at a high concentration factor condition can have high photothermal conversion efficiency. Simple periodic channels were ablated on stainless steel substrate to increase the absorptance. Based on the measured absorptance spectrum of this sample, the photothermal conversion coefficient can reach 0.87 if operated under the temperature of $1073 \mathrm{~K}$. 


\section{References}

1. Bermel P, Lee J, Joannopoulos JD, Celanovic I, Soljačić M. Selective Solar Absorbers. Annual Review of Heat Transfer. 2012;15:231-254

2. Moon J, Lu D, VanSanders B, Kim TK, Kong SD, Jin S, et al. High performance multi-scaled nanostructured spectrally selective coating for concentrating solar power. Nano Energy. 2014;8:238-246.

3. Cao F, McEnaney K, Chen G, Ren Z. A review of cermet-based spectrally selective solar absorbers. Energy \& Environmental Science. 2014;7(5):1615-1627.

4. Elsarrag E, Pernau H, Heuer J, Roshan N, Alhorr Y, Bartholomé $\mathrm{K}$. Spectrum splitting for efficient utilization of solar radiation: a novel photovoltaic-thermoelectric power generation system. Renewables: Wind, Water, and Solar. 2015;2:16.

5. Sakurai A, Tanikawa H, Yamada M. Computational design for a wide-angle cermet-based solar selective absorber for high temperature applications. Journal of Quantitative Spectroscopy and Radiative Transfer. 2014;132:80-89.

6. Kussmaul M, Mirtich MJ, Curren A. Ion beam treatment of potential space materials at the NASA Lewis Research Center. Surface and Coatings Technology. 1992;51(1-3):299-306.

7. Lampert CM. Heat mirror coatings for energy conserving windows. Solar Energy Materials. 1981;6(1):1-41.

8. Rinnerbauer V, Shen Y, Joannopoulos JD, Soljačić M, Schäffler F, Celanovic I. Superlattice photonic crystal as broadband solar absorber for high temperature operation. Optics Express. 2014;22(S7):A1895-A1906.

9. Rinnerbauer V, Lausecker E, Schäffler F, Reininger P, Strasser $\mathrm{G}$, Geil RD, et al. Nanoimprinted superlattice metallic photonic crystal as ultraselective solar absorber. Optica. 2015;2(8):743-746.

10. Sai H, Yugami H, Kanamori Y, Hane K. Solar selective absorbers based on two-dimensional W surface gratings with submicron periods for high-temperature photothermal conversion. Solar Energy Materials and Solar Cells. 2003;79(1):35-49.

11. Chen YB, Zhang ZM. Design of tungsten complex gratings for thermophotovoltaic radiators. Optics Communications. 2007;269(2):411-417.

12. Chou J, Yeng YX, Lenert A, Rinnerbauer V, Celanovic I, Soljačić $\mathrm{M}$, et al. Design of wide-angle selective absorbers/emitters with dielectric filled metallic photonic crystals for energy applications. Optics Express. 2014;22 Suppl 1:A144-154.
13. Arpin KA, Losego MD, Braun PV. Electrodeposited 3D Tungsten Photonic Crystals with Enhanced Thermal Stability. Chemistry of Materials. 2011;23(21):4783-4788.

14. Rinnerbauer V, Ndao S, Yeng YX, Senkevich JJ, Jensen $\mathrm{KV}$, Joannopoulos JD, et al. Large-area fabrication of high aspect ratio tantalum photonic crystals for hightemperature selective emitters. Journal of Vacuum Science \& Technology B, Nanotechnology and Microelectronics: Materials, Processing, Measurement, and Phenomena. 2013;31(1):011802.

15. Jiang D, Yang W, Tang A. A refractory selective solar absorber for high performance thermochemical steam reforming. Applied Energy. 2016;170:286-292.

16. Rinnerbauer V, Yeng YX, Chan WR, Senkevich JJ, Joannopoulos JD, Soljacic M, et al. High-temperature stability and selective thermal emission of polycrystalline tantalum photonic crystals. Optics Express. 2013;21(9):11482-11491.

17. National Renewable Energy Laboratory. Reference Solar Spectral Irradiance: Air Mass 1.5 Spectra. Available from: $<$ http://rredc.nrel.gov/solar/spectra/am1.5/>. Access in: $06 / 03 / 2018$

18. Hench JJ, Strakoš Z. The RCWA method - A case study with open questions and perspectives of algebraic computations. Electronic Transactions on Numerical Analysis. 2008;31:331357.

19. Li L. Fourier modal method for crossed anisotropic gratings with arbitrary permittivity and permeability tensors. Journal of Optics A: Pure and Applied Optics. 2003;5(4):345.

20. Li L. Use of Fourier series in the analysis of discontinuous periodic structures. Journal of the Optical Society of America A. 1996;13(9):1870-1876.

21. Li L. New formulation of the Fourier modal method for crossed surface-relief gratings. Journal of the Optical Society of America A. 1997;14(10):2758-2767.

22. Lalanne P, Morris GM. Highly improved convergence of the coupled-wave method for TM polarization. Journal of the Optical Society of America A. 1996;13(4):779-784.

23. Lalanne P. Improved formulation of the coupled-wave method for two-dimensional gratings. Journal of the Optical Society of America A. 1997;14(7):1592-1598.

24. RefractiveIndexDatabase.Info. Refractive INDEX. Available from: $<$ http://refractiveindex.info/>. Access in: 06/03/2018. 